\title{
Mechanical and Thermo-Physical Properties of Short Glass Fiber Reinforced Polybutylene Terephthalate upon Aging in Lubricant/Refrigerant Mixture
}

\author{
Guilherme Cybis Pereira ${ }^{a}$, Felipe Darabas Rzatki ${ }^{a}$, Luca Mazzaferro ${ }^{a}$, Daniel Maldonado Forin ${ }^{a}$, \\ Guilherme Mariz de Oliveira Barra \\ ${ }^{a}$ Department of Mechanical Engineering, Federal University of Santa Catarina - UFSC, Florianopolis, \\ SC, Brazil
}

Received: April 30, 2016; Revised: July 23, 2016; Accepted: September 04, 2016

\begin{abstract}
In this study, the relationship between structure and mechanical properties of aged PBT composite was investigated. Short glass fiber reinforced PBT composites were subjected to aging tests in environments typically found in hermetic compressors. Diffusion kinetics were addressed by a periodic control of samples weight, and samples aged in oil presented mass variation of $1.2-1.6 \%$, depending on test temperature. Most diffusion curves followed Fickian behavior, and the diffusion coefficient for each condition was obtained. Aging effect was investigated by 3-point bending tests, where samples aged at $180^{\circ} \mathrm{C}$ presented a significant reduction in all evaluated properties. Control environment induced minor variations, supposedly related to physical aging and increase in crystallinity. Imaging analyses through scanning electronic microscopy (SEM) technique allowed observation of the predominant fracture mechanism. Aged samples presented few or no localized plastic deformation at all, which is in accordance with the lower strain observed in mechanical tests. Differential scanning calorimetry (DSC) analyses proved degree of crystallinity increase for all aged samples, depending mainly on temperature of exposure. Infrared (FTIR) analysis proved the presence of oil absorbed in PBT chemical structure, and it was proposed a method for comparing degree of crystallinity, based on the absorbance bands ratio $\mathrm{A} 0\left(1458 \mathrm{~cm}^{-1}\right) / \mathrm{A} 0\left(956 \mathrm{~cm}^{-1}\right)$.
\end{abstract}

Keywords: Polybutylene terephthalate, aging, short glass fibers, degradation, diffusion.

\section{Introduction}

Polymeric materials are already widely used in the production of disposable and short-life-cycle products. Nowadays, however, there is a tendency of employing these materials in high-tech, structural and enduring applications, especially concerning the automotive and aerospace industry. The main advantages of polymers and its composites are many: firstly, they have a high strength/weight ratio, meaning that it is possible to conceive way lighter parts than those made of steel, for example, to attend similar stress requirements. Most polymers present high damping properties, are good thermal and electrical insulators and less susceptible to corrosion. In addition, they allow more freedom of design and ease of processability, usually accompanied with reduction of production costs.

On the other hand, the exposure of polymers to chemicaland thermally aggressive environments may lead to a gradual alteration of molecular conformation, chain size distribution and chemical composition, among others ${ }^{1,2}$. In other words, it occurs a time-dependent properties degradation, also known as aging. In some applications, the engineer will select a grade of polymer that does not react, or that have minimum interaction with the work environment; one example is the fabrication of fuel tanks made of high density polyethylene

* e-mail: guilherme.gui3038@gmail.com
(HDPE), a polymer that presents high chemical resistance against oil, diesel, alcohol and gasoline.

In different scenarios, though, other variables may be just as relevant for the material selection, for example, weight, mechanical properties, maximum work temperature, thermal conductivity, cost, and so forth ${ }^{3}$. For instance, the employment of polymers in refrigeration systems and hermetic compressors shows a huge potential in terms of overall efficiency improvement. In these particular cases, however, an important issue to be addressed is the chemical media in which the polymer will be located. Therefore, it is mandatory to characterize aging behavior and to understand mechanisms responsible for the polymer degradation in order to estimate properties loss in function of time, generating reliable data to aid the project of the component in a compatible way.

Degradation mechanisms of aromatic polyesters such as polyethylene terephthalate (PET) and polybutylene terephthalate (PBT) have been extensively studied over the past few decades ${ }^{4-10}$. Notwithstanding, just recently these polymers began to be aimed for engineering and structural applications. In these instances, it is common the addition of inorganic phases to the matrix in order to enhance mechanical properties. For these thermoplastic polymers, glass fibers are by far the most widely used type of reinforcements. Short glass fibers reinforced PBT is already used to produce some internal components in hermetic compressors, mainly due to 
its good temperature and chemical resistance, mechanical properties, thermal insulation and low cost. The potential of using PBT, however, is still limited by the lack of knowledge regarding its long-term behavior in contact with aggressive environments present inside hermetic compressors.

Cavestri $^{3}$, in 1993, was one of the first authors to study the compatibility of typical refrigerants and lubricants with engineering plastics. In the report prepared for the Air-Conditioning and Refrigeration Technology Institute (ARTI), it was tested up to 23 types of polymers in different environments, with the objective to create data referring to degradation of mechanical properties. It was found out that the most aggressive environment for PBT was the mixture of gaseous tetrafluoroethane and polyolester oil in high temperatures, and classified it as "worthy of further analyses". It was not studied, however, the mechanisms by which these polymers have their properties reduced, nor were addressed kinetic parameters of the diffusion process occurring during immersion tests. In addition, even though the author commented about the probable influence of reinforcements and fillers in the aging process, none of the 23 polymers tested was a composite.

Oréfice et al. ${ }^{11}$ affirms that the interface between fibers and matrix might be the preferable pathway for solution migration. Even if no chemical attack takes place, the presence of oil in the interface deteriorate reinforcement mechanisms by load transfer from polymer matrix to fibers. Mohd Ishak et al. ${ }^{12}$ studied the effects of hygrothermal aging on short glass fiber reinforced PBT, and discovered that a silane coupling agent helped prevent mechanical properties loss due to interface degradation. It was used in their study Fick's single-phase diffusion model to analyze the data. According to the authors, deviations from Fickian behavior are sometimes related to damage of the interface or resin degradation. Likewise, Bergeret ${ }^{13}$ have tested samples of glass-fiber reinforced PBT and PET exposed to aqueous environment. In that work, diffusion models were used to fit the experimental data. It was noticed an increase of acidic end-groups $(\mathrm{COOH})$ of aged samples due to hydrolysis, determined through a specific titration at high temperature. It was also detected a higher degree of crystallinity of the samples, measured by differential scanning calorimetry. It was observed mechanical properties reduction due to the matrix-fiber interface damage. Other authors ${ }^{11,14}$ have also proposed residual resistance tests as a method for evaluation of damage degree due to aging process.

Furthermore, it is well established that temperature plays one of the most important roles in aging phenomena. Ray ${ }^{15}$ proved the influence of the test temperature in aging phenomena of epoxy samples reinforced with glass and carbon fibers. The author stated that the increase of the temperature affects not only the kinetics of liquid absorption by the polymer, but also alters its mechanical resistance for a given swelling level. Hutchinson ${ }^{16}$ also affirms that the temperature has a strong influence, mainly due to the increasing molecular mobility, hence accelerating aging processes.

On the other hand, the use of reinforced thermoplastic polymers in the refrigeration industry is still limited by the lack of knowledge regarding their long-term behavior in contact with aggressive environments. The major factors influencing aging phenomena are temperature, time, affinity between polymer and environment, stress state, thickness of the sample and interface effects between two dissimilar phases (e.g. composites). There are several studies in the open literature concerning on aging of different kind of polymers subjected to aqueous environments or weathering $\left(\mathrm{O}_{2}, \mathrm{UV}\right.$ radiation, etc.). However, most of them are focused in either evaluating the influence of different variables on the resulting mechanical properties, or on diffusion kinetics and aging mechanisms assessments. In our knowledge there are no studies dealing the influence of aging tests on the mechanisms which PBT composites have their properties reduced, nor were addressed kinetic parameters of the diffusion process occurring during aging tests in environments typically found in hermetic compressors. Therefore, in this study the relationship between structure and mechanical properties of PBT composite upon aging in lubricant and refrigerant mixture used in hermetic compressors was investigated

\section{Materials and Methods}

\subsection{Materials}

The chemical media used in the tests consisted in a mixture of an ester-based lubricant oil and tetrafluoroethane refrigerant gas. The inert atmosphere used as a control environment was argon with $99.999 \%$ purity degree. The polymer tested was a polybutylene terephthalate commercial resin from Ticona (PBT CELANEX ${ }^{\circledR}$ grade 3300 ), reinforced with 30 wt. $\%$ of short glass fibers.

\subsection{Preparation of the samples}

The samples were produced via injection molding in an Allrounder 320S-Arburg machine, following the procedure described in the supplier datasheet. It was used an injection speed of $20 \mathrm{~mm} / \mathrm{s}$, pressure of 400 bar and temperature of $250^{\circ} \mathrm{C}$. The mold was kept at $60^{\circ} \mathrm{C}$ under $20 \mathrm{kN}$ of clamping force. The samples were produced according to the norm ASTM D790, which recommends the geometry for threepoint bending tests.

After injection, all samples passed through an annealing process at $180^{\circ} \mathrm{C}$ during two hours, in order to erase thermal history and eliminate possible residual stresses arising from the molding process $s^{3,17,18}$. For the measurement of initial weight values before aging, the samples were rinsed quickly in acetone, wiped dry with a paper tissue and then immediately weighed using a high precision analytical balance. 
For diffusivity and mechanical analyses, it was tested a minimum of five samples for each condition, while for DSC, TGA and FTIR it was used three samples per condition.

\subsection{Aging tests}

Aging tests were conducted based on ASTM D543, in a Carl-Roth autoclave with working capacity of $275 \mathrm{~mL}$, equipped with temperature and pressure control systems. In each round, five samples were placed into the autoclave and lubricant oil was added until total submersion of the test bars. The oil was previously dehumidified by vacuum ( $\sim 0.9$ mbar) during 3 hours. In sequence, refrigerant gas was injected until the interior pressure reached 3 bar, then the temperature was ramped to the pre-defined value and kept constant (uncertainty of $\pm 1^{\circ} \mathrm{C}$ ) using a commercial resistive heater apparatus connected to a N1200 Novus PID controller. The pressure had to be constantly adjusted (in order to maintain 3 bar) during the first two hours of test, due to effects of gas absorption by the oil and pressure increase due to temperature augment. After 28 days, the samples were moved to another recipient containing the same lubricant oil at $21^{\circ} \mathrm{C}$ during sixty minutes for cooling. Finally, samples were rinsed quickly in acetone, wiped dry with a paper tissue and then immediately measured their weight.

It was conceived three conditions of testing varying temperature inside the autoclave, 140,160 and $180^{\circ} \mathrm{C}$. In order to address pure temperature-induced effects, it was performed an additional aging process in an inert atmosphere, using a tubular oven filled with argon at $180^{\circ} \mathrm{C}$, during 14 days.

Both processes, in autoclave and in tubular oven, were previously validated with several repeated runs in order to obtain processes standard deviation. These values are already embedded to the values of error for the results presented in the next section.

\subsection{Diffusivity}

Oil absorption by the polymer was estimated by weight variation measurements, performed in a high precision analytical balance XS205 DualRange-Mettler Toledo. For immersion tests, it was fixed a duration of 28 days, which was chosen considering the results of previous tests, in order to guarantee that saturation would be achieved.

Based on similar studies, it was observed that the far most used approach for fluidic media absorption modeling was Fick's first law, applied for single free-phase diffusion ${ }^{1,11,12}$. Mass variation resultant from the media absorption in a given time $t,(\Delta \mathrm{m})$, can be expressed in terms of diffusion coefficient $D$ and saturation limit $(\Delta \mathrm{m})_{\infty}$, related in equations 1 and 2. According to this model, the mass gain due to solute absorption increases linearly with the square root of time, and gradually decelerate until equilibrium is reached.
For short times, where $D t / h^{2}<0.05$, it was applied the equation $1^{1,11,12}$ :

$$
\frac{(\Delta \mathrm{m}) t}{(\Delta \mathrm{m})_{\infty}}=\frac{4}{\sqrt{\pi}} \sqrt{\frac{D t}{h^{2}}}
$$

where $h$ is the sample thickness. For longer times $\left(D t / h^{2}\right.$ $>0.05)$, the most adequate mathematical expression is ${ }^{1,12}$ :

$$
\frac{(\Delta \mathrm{m}) t}{(\Delta \mathrm{m})_{\infty}} \approx 1-\frac{8}{\pi^{2}} \exp \left(-\frac{\pi^{2} D t}{h^{2}}\right)
$$

The diffusion coefficient is proportional to the slope of the linear portion of the normalized $(\Delta \mathrm{m})_{t} /(\Delta \mathrm{m})_{\infty} \mathrm{vs} / \frac{\sqrt{t}}{h}$ curve, and was obtained from equation 1 . The saturation limit was taken as the maximum value of mass variation measurements.

\subsection{Mechanical analysis}

The mechanical analysis was done following the threepoint bending method described in ASTM D790, using an EMIC DL2000 universal testing machine. The span length was adjusted to $60.8 \mathrm{~mm}$, and the crosshead speed was kept constant at $1.5 \mathrm{~mm} / \mathrm{min}$ until the breaking of the sample. Values of stress and strain were calculated by equations 3 and 4 , respectively.

$$
\begin{gathered}
\sigma_{f}=\frac{3 F L}{2 l h^{2}} \\
\varepsilon=\frac{6 \delta h}{L^{2}}
\end{gathered}
$$

where $\sigma_{f}$ is the flexural resistance, $\varepsilon$ is the strain, $F$ is the applied force, $\delta$ is the crosshead displacement, $L$ is the span length and $l$ and $h$ represent sample's width and thickness, respectively.

Values of flexural resistance and total strain were calculated in form of an arithmetic average between tested samples. In order to correct total strain values for initial slipping and clamping effects, it was necessary to use the "toe compensation" technique described in ASTM D790. Flexural modulus was assumed as the slope of the straight fitting of the linear elastic part of the curves (from $0.2 \%$ to $0.4 \%$ ).

\subsection{Fracture surface characterization}

Scanning electronic microscopy (SEM) was done in a Jeol-JSM 6390LV microscope. It was analyzed the fracture surface morphology of the samples previously subjected to three-point bending tests. Samples were cut about $6 \mathrm{~mm}$ below their fracture surface, bonded with a carbon adhesive tape to support stubs and afterwards covered with a gold coating. 


\subsection{Thermal analyses}

Differential scanning calorimetry (DSC) and thermogravimetry (TG) analyses were carried out in a DSC-50 - Shimadzu equipment and in a simultaneous thermal analyzer Jupter F3-Netzsch, respectively.

Thermal profile of DSC runs consisted in heating from $25^{\circ} \mathrm{C}$ to $280^{\circ} \mathrm{C}$ with a $10^{\circ} \mathrm{C} / \mathrm{min}$ rate and $50 \mathrm{~mL} / \mathrm{min}$ nitrogen flux. It was used sealed aluminum crucibles, and samples mass of about $14 \mathrm{mg}$. Crystallinity degree was calculated from equation 5 , based on a theoretical $100 \%$ crystalline PBT heat of fusion of $140 \mathrm{~J} / \mathrm{mol}^{19,20}$.

$\%$ Crystallinity $=\frac{\Delta H_{f}-\Delta H_{c}}{\Delta H_{f}^{\circ}} \times 100 \%$

where $\Delta \mathrm{H}_{f}$ is the measured heat of fusion, $\Delta \mathrm{H}_{c}$ is the cold crystallization enthalpy and $\Delta \mathrm{H}_{f}$ is the $100 \%$ crystalline heat of fusion.

TG isothermal runs were done in order to study thermal stability of the polymer. Isothermal temperature was $260^{\circ} \mathrm{C}$, maintained for 50 hours, and the atmosphere inside the chamber consisted of argon with a $70 \mathrm{~mL} / \mathrm{min}$ flow.

\subsection{Spectroscopy analysis}

Fourier transform infrared spectroscopy (FTIR) analysis was performed in a Tensor 27 - Bruker equipment using the Attenuated Total Reflectance (ATR) method. It was used a ZnSe crystal with depth of penetration of $2.04 \mu \mathrm{m}$. The spectra were acquired from an average of 32 scans with a 4 $\mathrm{cm}^{-1}$ resolution, ranging from 4000 to $600 \mathrm{~cm}^{-1}$. Transmittance data were converted in absorbance by the relation $[\mathrm{A}=$ $\log (\mathrm{T})]$, followed by the spectrum intensity normalization based on a reference band in $1408 \mathrm{~cm}^{-1}$, which refers to $\mathrm{C}-\mathrm{C}$ stretch of the PBT aromatic ring. FTIR analyses were performed in several time span during the aging tests, in order to analyze the variation in crystallinity-related bands. The ratio between $1458 \mathrm{~cm}^{-1}$ (crystalline phase) and 956 $\mathrm{cm}^{-1}$ (amorphous phase) bands was chosen to represent the degree of crystallinity index, which allowed quantitative comparison among samples ${ }^{21,22}$.

\section{Results and Discussion}

\subsection{Weight variation}

After aging tests of samples in autoclave, it was observed an increase ranging from 1.2 to $1.6 \mathrm{wt} . \%$, depending on test temperature. This variation is assigned mainly to oil absorption by the polymer. Control samples presented a 0.2 wt.\% loss, due probably to humidity extraction. Diffusion coefficient and maximum oil absorption results can be found in Table 1.
Table 1: Diffusion coefficient and maximum oil absorption results.

\begin{tabular}{lcc}
\hline Temperature $\left({ }^{\circ} \mathrm{C}\right)$ & $\begin{array}{c}\text { Diffusion coefficient } \\
\left(\mathrm{cm}^{2} / \mathrm{s}\right) \times 10^{-8}\end{array}$ & $\begin{array}{c}\text { Max. oil absorption } \\
(\text { wt. } \%)\end{array}$ \\
\hline 140 & $2.81 \pm 0.62$ & $1.23 \pm 0.05$ \\
160 & $3.71 \pm 0.56$ & $1.42 \pm 0.05$ \\
180 & $6.78 \pm 0.54$ & $1.56 \pm 0.05$ \\
\hline
\end{tabular}

By diffusion coefficient results, it appears that temperature accelerates diffusion kinetics exponentially, and hence promotes a more rapidly saturation of oil inside the polymer, which is confirmed in Figure 1.

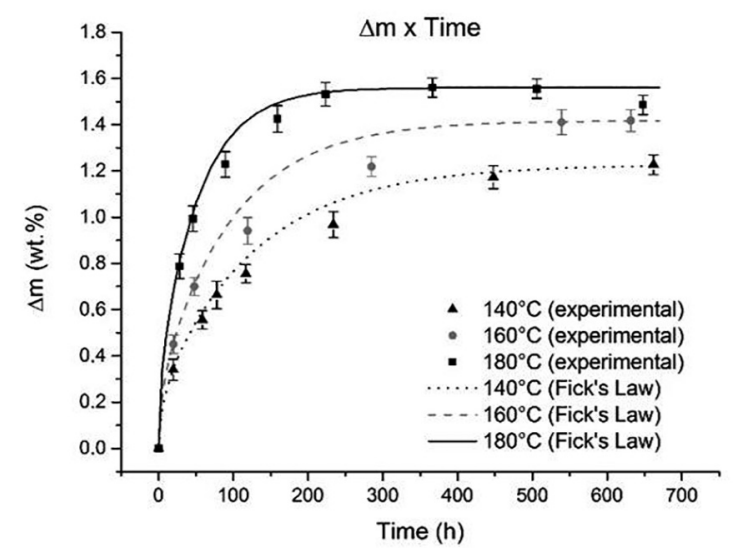

Figure 1: Oil uptake vs aging time for three temperature conditions. The lines correspond to theoretical values calculated by Fick's Law equations.

The experimental data shows good fit with theoretical values calculated by equations 1 and 2 , except in the intermediate region, of about $70-85 \%$ of the maximum point of the curves. One possible explanation is the occurrence of solubilization and extraction of oligomers by the environment, which can also explain the inversion in the curve slope at $180^{\circ} \mathrm{C}$ after 500 hours.

Some authors reported the independence between saturation limit and temperature ${ }^{12}$, while others have found that this value could vary ${ }^{23-25}$. In the present study, the maximum oil absorption appears to vary linearly with temperature (Table 1). Nevertheless, it is necessary to state that 28 days might not have been time enough to allow saturation of the system, and that oil absorption measurements could reach the same value over a long period of time, independently of the test temperature.

\subsection{Three-point bending tests}

It was compared the results of three-point bending tests for five conditions: virgin, aged in three different temperatures and a control group, all presented in Table 2. One can notice that all samples aged in autoclave presented a poorer flexural strength, tangent modulus and maximum strain than virgin 
Table 2: Mechanical properties in bending test.

\begin{tabular}{lccc}
\hline Condition & $\sigma_{\mathrm{f}}(\mathrm{MPa})$ & $\mathrm{E}(\mathrm{GPa})$ & $\varepsilon_{\text {max. }}(\mathrm{mm} / \mathrm{mm})$ \\
\hline Virgin & $181.8 \pm 3.4$ & $8.21 \pm 0.13$ & $(3.23 \pm 0.12) \%$ \\
Aged $\left(140^{\circ} \mathrm{C}\right)^{1}$ & $170.9 \pm 5.9$ & $6.91 \pm 0.43$ & $(3.33 \pm 0.16) \%$ \\
Aged $\left(160^{\circ} \mathrm{C}\right)^{1}$ & $152.2 \pm 4.9$ & $6.96 \pm 0.37$ & $(2.87 \pm 0.11) \%$ \\
Aged $\left(180^{\circ} \mathrm{C}\right)^{1}$ & $96.3 \pm 5.6$ & $6.66 \pm 0.48$ & $(1.93 \pm 0.14) \%$ \\
Control $\left(180^{\circ} \mathrm{C}\right)^{2}$ & $197.6 \pm 6.7$ & $8.62 \pm 0.21$ & $(3.18 \pm 0.19) \%$ \\
\hline
\end{tabular}

${ }^{1}$ Aged for 28 days in lubricant/refrigerant mixture. ${ }^{2}$ Aged for 14 days in inert atmosphere.

samples. Aging temperature affected considerably strength and maximum strain values, while it did not change significantly the elastic modulus.

On the other hand, samples that passed through the control aging process in an inert atmosphere presented higher properties even than virgin samples (see Figure 2), indicating that the degradation of the polymer properties occurred mainly due to interaction with the chemical media, instead of solely high temperature exposure. Nevertheless, temperature is likely to hasten aging process by accelerating possible chemical reactions and also due to the increase of free volume between polymer molecules, which leads to a higher oil diffusion rate through the material ${ }^{15,16}$.

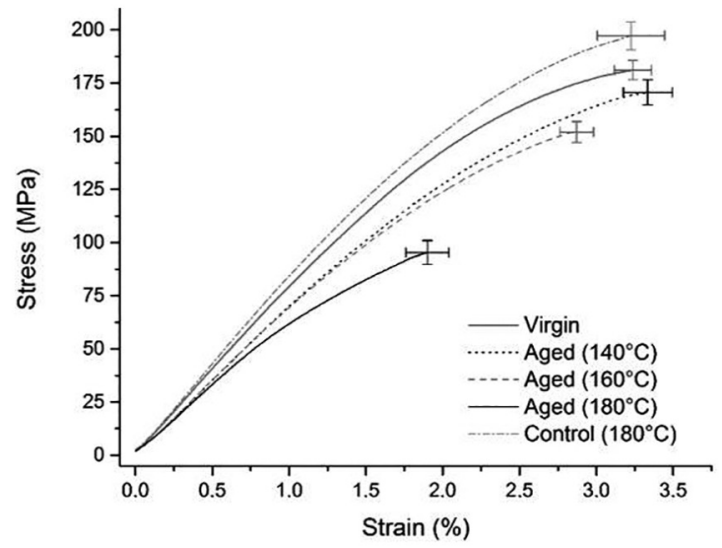

Figure 2: Plot of three-point bending test curves.

One possible explanation for the presented results is that the oil inside the polymer acted as a plasticizer, hence decreasing modulus and strength. It can also be conjectured that some kind of degradation reaction occurred, lowering molecular weight by breaking polymer chains, which would cause a decay in mechanical properties. A third assumption, referenced by Oréfice et al. ${ }^{11}$, is that the interface between fibers and matrix is the preferable pathway for solution migration. Even if no chemical attack takes place, the presence of oil in the interface deteriorates reinforcement mechanisms by load transfer from the polymer matrix to the fibers. All three mentioned effects could be acting simultaneously to cause the properties drop. Moreover, control samples may have presented enhanced mechanical properties due to augmented crystallinity degree and physical aging.

\subsection{Fracture surface characterization}

SEM images allowed the characterization of the fracture surface morphology. It was observed that the predominant fracture mechanism consisted on the formation of a region with localized plastic deformation and fiber debonding, followed by a bigger region showing characteristics of fragile cleavage rupture (Figure 3).

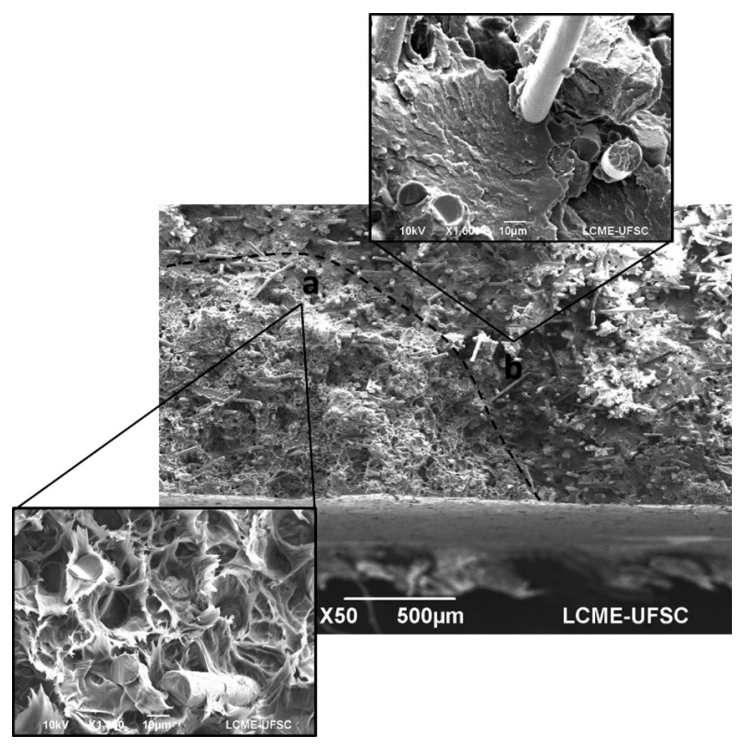

Figure 3: SEM micrograph (x50) of virgin sample, showing the regions of plastic deformation (a) and fragile rupture (b) in detail $(\mathrm{x} 1,000)$.

Both virgin and control samples showed similar behavior, with a localized plastic deformation region covering about $1 / 6$ of the fracture surface total area. Aged samples, on the other hand, presented few or no localized plastic deformation at all, which is in accordance with the lower strain observed in 3 -point bending tests. This clear reduction in matrix ductility is likely to be caused by some kind of degradation reaction of PBT macromolecules, leading to an overall mechanical properties decrease.

Moreover, it was analyzed the quality of the interfaces between fibers and matrix (Figure 4). It was observed that aged samples presented some gaps between fibers and matrix, what could indicate poorer interfacial bonding than virgin and control samples. This may be related to the presence 


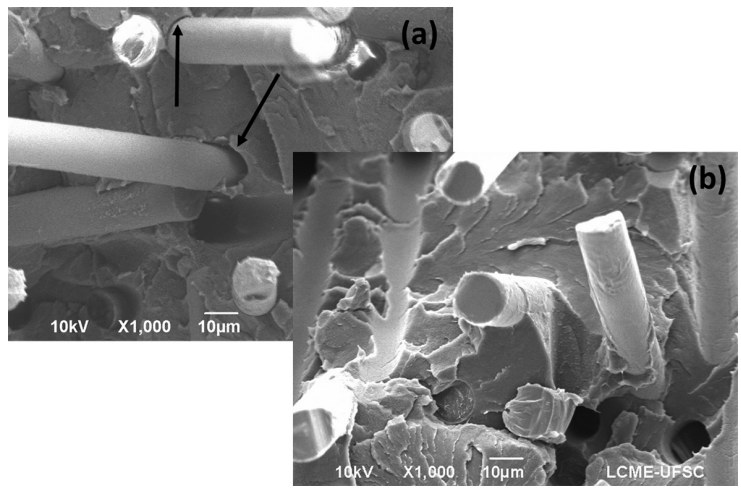

Figure 4: SEM micrograph $(x 1,000)$ of aged (a) and virgin $(b)$ samples, indicating the first poorer interfacial bonding between fibers and matrix.

of oil diffused through the interfaces. This phenomenon would also contribute to the reduction of the mechanical properties, since it deteriorates reinforcement mechanisms by load transfer from polymer matrix to fibers ${ }^{11}$.

\subsection{DSC/TG analyses}

DSC thermograms are consistent with previous literature results from $\mathrm{PBT}^{20}$. The higher the aging temperature, the more intense and shifted to higher temperatures is the secondary melting peak. This second peak appears on the left side of the original one in the thermogram in Figure 5. This new melting peak is also observed in the control samples DSC thermogram.

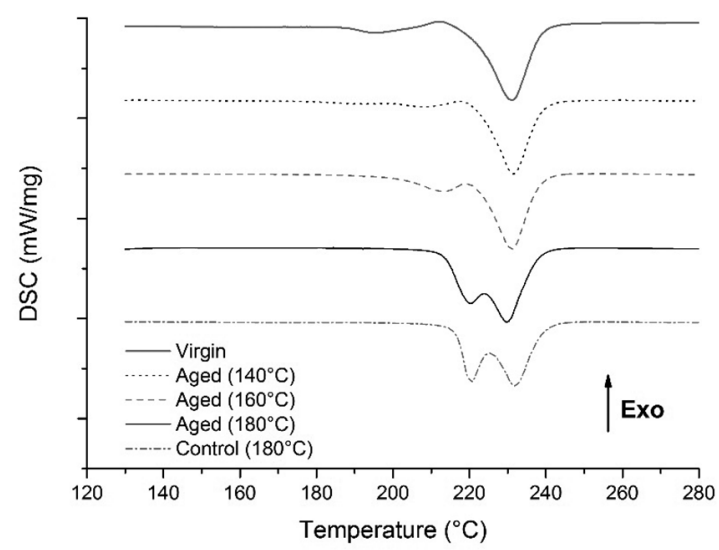

Figure 5: DSC curves showing melting peaks of PBT samples.

It is well known from literature ${ }^{19,26}$ that multiple peaks phenomenon is usual from PBT calorimetry analyses and can be attributed mainly to thermal history of the polymer. According to $\mathrm{Hobbs}^{27}$, the appearance of a second peak below the original peak can occur due to reorganization of crystalline structure or recrystallization of previously amorphous material during thermal treatment. The reason why these new crystals have lower melting points ones is that they are formed in lower temperatures and therefore they organize in thinner lamellae structures, which are less thermodynamically stable.

From Table 3 it is possible to notice that the lower peak melting point is dependable on the aging temperature, as well as the degree of crystallinity. Moreover, control samples showed a similar behavior to those aged at $180^{\circ} \mathrm{C}$, which indicates that the crystalline structure may not suffer much influence by the environment. The slightly lower crystallinity degree observed in control samples could be consequence of the short duration of thermal treatment (14 days against 28 days for aged samples). However, it could also be a result of a chemi-crystallization process during autoclave aging, induced by the break of amorphous chains and subsequent crystallization of the smaller and more mobile segments ${ }^{6,7}$.

Isothermal runs at $260^{\circ} \mathrm{C}$ revealed an interest degradation behavior of the samples aged at $180^{\circ} \mathrm{C}$. As it is shown in Figure 6 , mass loss ratios after 600 or so minutes of test are really similar for the three conditions. In the earlier stages, though, the aged sample loses mass much more rapidly than the other two. At 600 minutes of test, the mass variation gap between them is almost 6 wt.\%. As the weight gained by the polymer by oil absorption is about $1.6 \mathrm{wt} . \%$, this means that, either the oil absorption measurements were not accurate, or there are other compounds of lower molecular weight being degraded at early stages of the TG runs. The equipment balance error is $0.0025 \mathrm{wt} . \%$.

\subsection{FTIR spectroscopy}

FTIR spectra of aged and virgin PBT are illustrated in Figure 7. Typical IR bands of non-aged PBT appear at wavenumbers of $1707 \mathrm{~cm}^{-1}$ (carbonyl stretch), $1456 \mathrm{~cm}^{-1}(\mathrm{C}-\mathrm{H}$ bending in $\mathrm{CH}_{2}$ group), 1407 and $1018 \mathrm{~cm}^{-1}$ (aromatic ring), $1241 \mathrm{~cm}^{-1}$ (CO-O stretch in esters), $1115 \mathrm{~cm}^{-1}\left(\mathrm{O}-\mathrm{CH}_{2}\right)$, and $723 \mathrm{~cm}^{-1}$ (aromatic $\mathrm{C}-\mathrm{H}$ bending) ${ }^{19,28}$. As shown in Figure 7 , no major changes between different PBT spectra can be noticed. In aged samples, however, it was detected the rise of a band around $1160 \mathrm{~cm}^{-1}$ and the appearance of a shoulder approximately at $1740 \mathrm{~cm}^{-1}$. After analyzing samples of pure ester oil droplets, it was able to attribute these changes to the presence of the oil in PBT structure. The ester oil has two main absorption bands, located exactly at 1157 and 1738 $\mathrm{cm}^{-1}$, which are related to $\mathrm{C}-\mathrm{O}-\mathrm{C}$ and $\mathrm{C}=\mathrm{O}$ groups axial vibrations, respectively.

Deconvolution methods were employed in order to obtain the single contribution of the bands of interest to the integrated absorbance. Crystallinity-related bands in 1458 $\mathrm{cm}^{-1}$ (crystalline phase) and $956 \mathrm{~cm}^{-1}$ (amorphous phase) were integrated and then used to calculate the degree of crystallinity index, stipulated as the dichroic ratio $\mathrm{A}_{0}(1458$ $\left.\mathrm{cm}^{-1}\right) / \mathrm{A}_{0}\left(956 \mathrm{~cm}^{-1}\right)$. 
Table 3: Melting point and crystallinity degree of the samples measured by DSC.

\begin{tabular}{lccccc}
\hline \multirow{2}{*}{ Condition } & \multicolumn{2}{c}{$\mathrm{T}_{\mathrm{m}}\left({ }^{\circ} \mathrm{C}\right)$} & \multicolumn{2}{c}{ Crystallinity degree } \\
& Lower peak & Original peak & Lower peak & Original peak & Overall \\
\hline Virgin & $195.5 \pm 0.7$ & $231.7 \pm 0.6$ & $(2.3 \pm 1.1) \%$ & $(23.8 \pm 1.6) \%$ & $(26.1 \pm 2.0) \%$ \\
Aged $\left(140^{\circ} \mathrm{C}\right)$ & $208.2 \pm 0.5$ & $231.2 \pm 1.0$ & $(3.9 \pm 1.3) \%$ & $(23.3 \pm 3.3) \%$ & $(27.3 \pm 4.5) \%$ \\
Aged $\left(160^{\circ} \mathrm{C}\right)$ & $214.1 \pm 1.0$ & $231.1 \pm 0.5$ & $(9.6 \pm 1.1) \%$ & $(22.5 \pm 0.8) \%$ & $(32.1 \pm 1.9) \%$ \\
Aged $\left(180^{\circ} \mathrm{C}\right)$ & $219.9 \pm 0.5$ & $229.7 \pm 0.9$ & $(12.6 \pm 1.1) \%$ & $(22.8 \pm 1.6) \%$ & $(35.4 \pm 2.6) \%$ \\
Control $\left(180^{\circ} \mathrm{C}\right)$ & $220,1 \pm 0,8$ & $231,5 \pm 0,8$ & $(11,8 \pm 0,9) \%$ & $(20,7 \pm 1,7) \%$ & $(32,5 \pm 2,6) \%$ \\
\hline
\end{tabular}

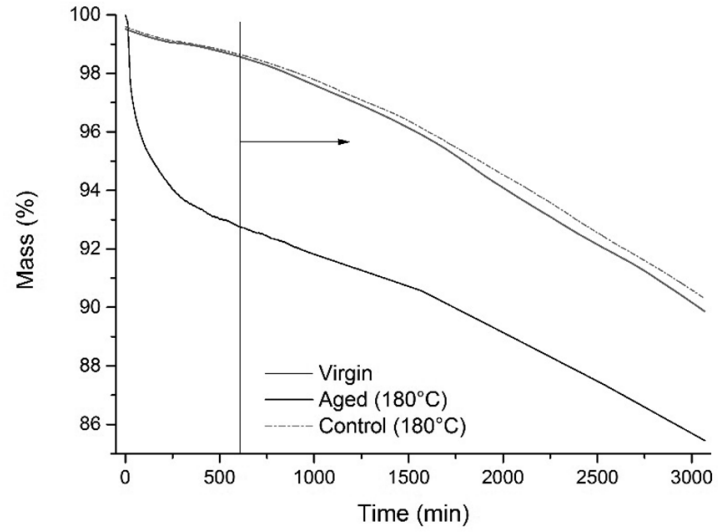

Figure 6: TG isothermal curves of mass loss in function of time, at $260^{\circ} \mathrm{C}$.

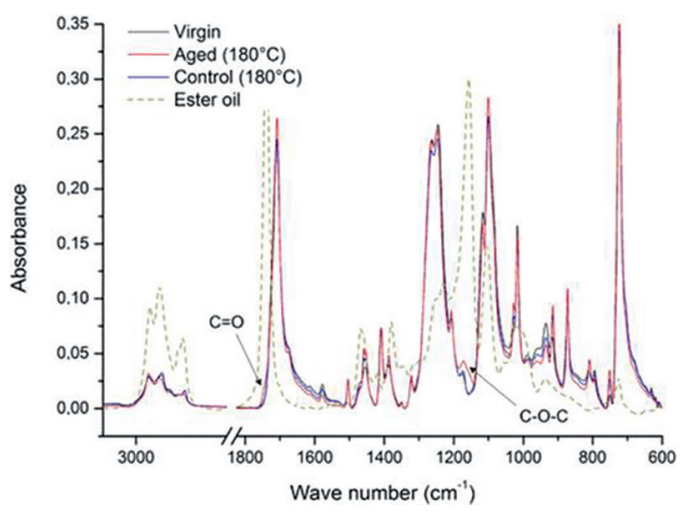

Figure 7: Comparison of PBT and ester oil FTIR spectra.

By periodically measuring this ratio, it was possible to plot the graph in Figure 8, which shows the developing of the crystallinity index during aging tests. This index, though not an absolute value, aims to represent the increase of the crystallinity degree in function of aging time. One can observe that the residual crystallization in aging tests is greatly dependent not only on temperature, as it was shown in DSC analyses, but also on time, proving that it is a phenomenon driven by molecular mobility. It is expected that the degree of crystallinity eventually stabilizes, but it is not possible to state that it has, within 28 days.

As stated before, there was no evidence of any important structural change that could indicate the occurrence of some

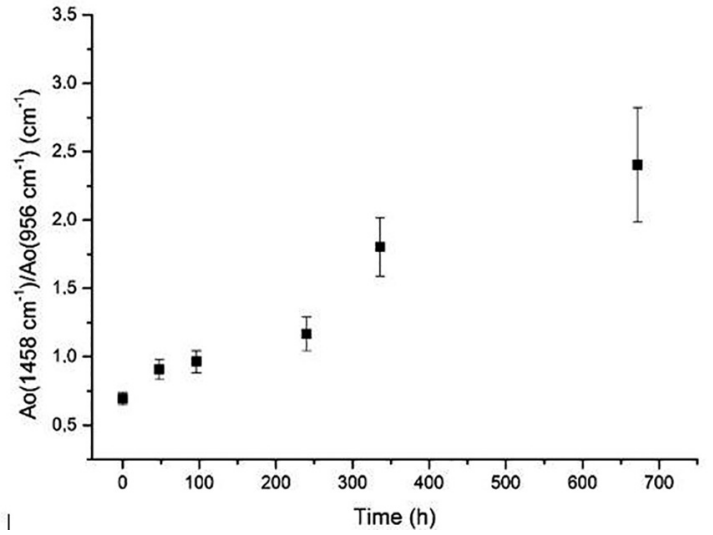

Figure 8: Developing of $A_{0}\left(1458 \mathrm{~cm}^{-1}\right) / \mathrm{A} 0\left(956 \mathrm{~cm}^{-1}\right)$ ratio, relating to the increase in crystallinity during aging tests.

degradation process during aging. A hypothesis is that the active degradation mechanisms do not generate any new end-group in PBT structure. This is the case, for example, of transesterification reactions, in which simply occurs an exchange between radicals attached to ester and alcohols groups of different compounds. It would be possible, therefore, the occurrence of chain scission reactions with exchange of ester groups between the PBT and oil, with no significant change in terminal groups signals. The verification of this hypothesis will be subject of a future work in the area.

\section{Conclusions}

It was proved the interaction between PBT composite and the ester oil/tetrafluoroethane mixture. It was observed absorption of oil by the polymer through weight measurements, TG analysis and FTIR spectra. Diffusion behavior was successfully fitted with Fick's first law equations. A phenomenon that is not predicted by Fickian behavior, however, is the solubilization and extraction of oligomers, which is likely to occur in high temperatures. Therefore, it can induce some deviations from the theoretical data. It was also noticed that aging led to a deterioration of mechanical properties of the polymer, where samples aged at $180^{\circ} \mathrm{C}$ were the most affected. Control samples, on the other hand, presented an even enhanced mechanical performance, probably due to physical aging and increase in crystallinity. By DSC 
analysis, it was evidenced the increase of the crystallinity degree. This residual crystallization is characterized by the formation of thinner lamellae crystals, and consequently lower melting temperature than original ones. SEM images revealed the predominant fracture mechanism, consisting on the formation of a region with localized plastic deformation and fiber debonding, followed by a bigger region showing characteristics of fragile cleavage rupture. It was not possible, via the characterization analyses performed in this study, to identify the actual cause of the observed mechanical properties degradation. It was hypothesized the occurrence of degradation due to transesterification reactions between PBT the ester oil. This hypothesis will be subject of further studies, but it is reinforced by thermogravimetry analyses (TGA), which show a steeply mass loss rate in the early stages of thermal degradation.

\section{Acknowledgements}

The authors would like to thank the FAPESC, CNPq and Embraco for providing financial and technical assistance. Also, we thank Celanex/Ticona for kindly providing the PBT resin used in the experiments.

\section{References}

1. Lagouvardos PE, Pissis P, Kyritsis A, Daoukaki D. Water sorption and water-induced molecular mobility in dental composite resins. Journal of Materials Science: Materials in Medicine. 2003;14(9):753-759.

2. Affolter S. Long-term behaviour of thermoplastic materials. Buchs: Interstate University NTB; 2008.

3. Cavestri RC. Compatibility of refrigerants and lubricants with engineering plastics. Dublin: Imagination Resources; 1993.

4. Maxwell AS, Broughton WR, Dean G, Sims GD. Review of accelerated ageing methods and lifetime prediction techniques for polymeric materials. Teddington: National Physical Laboratory; 2005.

5. Eipper A, Hanley S, Natarajan KM, Reinfrank K. Hydrolytically Stable PBT: Testing Gets Tougher. Kunststoffe International. 2008;5:64-66.

6. Allen NS, Edge M, Mohammadian M. Hydrolytic degradation of poly(ethylene terephthalate): Importance of chain scission versus crystallinity. European Polymer Journal. 1991;27(12):1373-1378.

7. Ballara A, Verdu J. Physical aspects of the hydrolysis of polyethylene terephthalate. Polymer Degradation and Stability. 1989;26(4):361-374.

8. Beyler CL, Hirschler MM. Thermal decomposition of polymers. In: DiNenno P, ed. SFPE Handbook of Fire Protection Engineering. 3rd ed. Quincy: Springer; 2002.

9. Montaudo G, Puglisi C, Samperi F. Primary thermal degradation mechanisms of PET and PBT. Polymer Degradation and Stability. 1993;42(1):13-28.
10. Jang S, Yang EK, Jin SI, Cho YD, Choe EK, Park CR. Characterization of thermal degradation of polytrimethylene terephthalate by MALDI-TOF Mass Spectrometry. Bulletin of the Korean Chemical Society. 2012;33(3):833-838.

11. Oréfice R, West J, Latorre G, Hench L, Brennan A. Effect of long-term in vitro testing on the properties of bioactive glasspolysulfone composites. Biomacromolecules. 2010;11(3):657-665.

12. Mohd Ishak ZA, Ariffin AA, Senawi R. Effects of hygrothermal aging and a silane coupling agent on the tensile properties of injection molded short glass fiber reinforced poly(butylene terephthalate) composites. European Polymer Journal. 2001;37(8):1635-1647.

13. Bergeret A, Ferry L, Ienny P. Influence of the fibre/ matrix interface on ageing mechanisms of glass fibre reinforced thermoplastic composites (PA-6,6, PET, PBT) in a hygrothermal environment. Polymer Degradation and Stability. 2009;94(9):1315-1324.

14. Hulme A, Cooper J. Life prediction of polymers for industry. Sealing Technology. 2012;2012(9):8-12.

15. Ray BC. Temperature effect during humid ageing on interfaces of glass and carbon fibers reinforced epoxy composites. Journal of Colloid and Interface Science. 2006;298(1):111-117.

16. Hutchinson JM. Physical aging of polymers. Progress in Polymer Science. 1995;20(4):703-760.

17. Kim J, Nichols ME, Robertson RE. The annealing and thermal analysis of poly(butylene terephthalate). Journal of Polymer Science Part B: Polymer Physics. 1994;32(5):887-899.

18. Yeh JT, Runt J. Fatigue crack-propagation in annealed poly(butylene terephthalate). Journal of Materials Science. 1989;24(7):2637-2642.

19. Deshmukh GS, Pathak SU, Peshwe DR, Ekhe JD. Effect of uncoated calcium carbonate and stearic acid coated calcium carbonate on mechanical, thermal and structural properties of poly(butylene terephthalate) (PBT)/calcium carbonate composites. Bulletin of Materials Science. 2010;33(3):277- 284.

20. Wu C. Preparation and characterization of an aromatic polyester/ polyaniline composite and its improved counterpart. Express Polymer Letters. 2012;6(6):465-475.

21. Holland-Moritz K, Siesler HW. Characterization of deformation phenomena in polymers by rapid-scanning fourier transform IR (FTIR) spectroscopy and mechanical measurements. Polymer Bulletin. 1981;4(3):166-170.

22. Kalkar AK, Siesler HW, Pfeifer F, Wadekar SA. Molecular orientation and relaxation in poly(butylene terephthalate)/ polycarbonate blends. Polymer. 2003;44(23):7251-7264.

23. Mousa A, Ishiaku US, Mohd Ishak ZA. Oil Resistance of Dynamically Vulcanized Poly(Vinyl Chloride)/Nitrile Butadiene Rubber Thermoplastic Elastomers. Polymer Bulletin. 2005;53(3):203-212.

24. Peniche C, Cohen ME, Vázquez B, Román JS. Water sorption of flexible networks based on 2-hydroxyethyl methacrylatetriethylenglycol dimethacrylate copolymers. Polymer. 1996;38(24):5977- 5982. 
25. Royer JR, Desimone JM, Khan SA. Carbon dioxide-induced swelling of poly(dimethylsiloxane). Macromolecules. 1999;32(26):8965-8973.

26. Kong Y, Hay JN. The enthalpy of fusion and degree of crystallinity of polymers as measured by DSC. European Polymer Journal. 2003;39(8):1721-1727.
27. Hobbs SY, Pratt CF. Multiple melting in poly(butylene terephthalate). Polymer. 1975;16(6):462-464.

28. Xiao J, Hu Y, Yang L, Cai Y, Song L, Chen Z, et al. Fire retardant synergism between melamine and triphenyl phosphate in poly(butylene terephthalate). Polymer Degradation and Stability. 2006;91(9):2093-2100. 